\title{
Le programme spécial de recherche et de formation concernant les maladies tropicales (TDR) : un point de vue
}

\author{
René Le Berre
}

\section{Le Tropical Diseases} Research Programme (TDR) est un programme de l'Organisation mondiale de la santé (OMS) ayant pour but de stimuler les recherches sur les maladies tropicales et de renforcer la formation des acteurs de la lutte contre ces maladies. L'un de ses responsables présente ce programme, son organisation, ses moyens et ses objectifs.
TIRÉS A PART

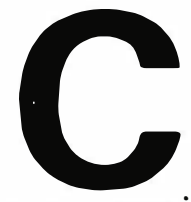

urieusement, c'est la rencontre d'une bactérie et d'un vertébré qui donna naissance à un programme hautement parasitologique: le Tropical Diseases Research Programme (TDR).

En effet, la découverte, en 1971, de la possibilité d'infecter le tatou par Mycobacterium le prae provoqua l'association de plusieurs équipes de chercheurs qui devait donner naissance, en 1974, au groupe de travail scientifique sur l'immunologie de la lèpre, germe initial du Programme. Sur un plan plus politique, l'Assemblée mondiale de la santé décidait, en mai 1974, d'intensifier les activités de l'Organisation mondiale de la santé (OMS) dans le domaine des maladies tropicales, de définir les priorités en tenant compte des besoins des pays en développement et d'amplifier la coopération de l'Organisation avec des institutions nationales, en vue de coordonner les efforts.

Cette décision, qui fut l'objet d'une résolution de l'Assemblée mondiale de la santé, provoqua l'association du Programme des Nations unies pour le développement (PNUD) de la Banque mondiale et de l'Organisation mondiale de la santé (OMS) dans une aventure commune, le TDR.

Le mandat de TDR, exécuté par l'OMS, est double: (1) mettre au point de nouvelles méthodes de prévention, de diagnostic, de traitement et de lutte antivectorielle adaptées à six maladies tropicales et aux pays qui en sont affectés; (2) renforcer, grâce à la formation de leurs chercheurs et aux formes diverses de soutien à leurs institutions spécialisées, le potentiel de recherche des pays les plus affectés par les maladies tropicales.

Les groupes de maladies initialement sélectionnés furent - et sont toujours - les suivants : paludisme, schistosomiase, filarioses, y compris l'onchocercose, trypanosomiases (maladie du sommeil et maladie de Chagas), leishmanioses et, bien entendu, lèpre.

Afin de couvrir ses deux objectifs, TDR est organisé de la manière suivante.

\section{Les comités d'orientation scientifique}

Ces comités d'orientation scientifique - traduction littérale et inélégante de l'anglais (steering committees : SC) - sont responsables de la recherche et du développement des méthodes et outils de prévention, de diagnostic, de traitement et d'évaluation pour chacun des groupes de maladies et pour des thèmes transversaux tels que la recherche socioéconomique et la lutte biologique contre les vecteurs. Chaque spécialiste d'un champ de recherche déterminé est, potentiellement, membre du comité correspondant. 
Tableau I

GROUPES DE TRAVAIL SCIENTIFIQUES ET COMITÉS D’ORIENTATION EN FONCTION EN 1986

\begin{tabular}{|c|c|c|c|}
\hline Composante & $\begin{array}{l}\text { Groupe de travail } \\
\text { scientifique }\end{array}$ & Comité d'orientation & Principales lignes de recherche \\
\hline Paludisme & $\begin{array}{l}\text { Chimiothérapie } \\
\text { Immunologie } \\
\text { Recherche appli- } \\
\text { quée de terrain }\end{array}$ & $\begin{array}{l}\text { CHEMAL } \\
\text { IMMAL } \\
\text { FIELDMAL }\end{array}$ & $\begin{array}{l}\text { Nouveaux médicaments, meilleur emploi des médica- } \\
\text { ments existants. } \\
\text { Vaccins, tests immunodiagnostiques. } \\
\text { Amélioration de la lutte sur le terrain. }\end{array}$ \\
\hline Schistosomiase & Schistosomiase & Schistosomiase & $\begin{array}{l}\text { Recherche fondamentale, y compris des études sur le } \\
\text { mode d'action des médicaments. } \\
\text { Études immunopathologiques. } \\
\text { Tests diagnostiques. } \\
\text { Études épidémiologiques. }\end{array}$ \\
\hline Filariose & Filariose & Filariose & $\begin{array}{l}\text { Nouveaux médicaments, meilleur emploi des médica- } \\
\text { ments existants. } \\
\text { Études sur l'immunopathologie. } \\
\text { Tests diagnostiques. } \\
\text { Études épidémiologiques. }\end{array}$ \\
\hline $\begin{array}{l}\text { Trypanosomiases } \\
\text { africaines }\end{array}$ & $\begin{array}{l}\text { Trypanosomiases } \\
\text { africaines }\end{array}$ & $\begin{array}{l}\text { Épidémiologie, biologie des } \\
\text { vecteurs et lutte antivectorielle } \\
\text { (EPIAF) } \\
\text { Chimiothérapie et immunolo- } \\
\text { gie et anatomopathologie } \\
\text { (CHEMAF/IMMAF) }\end{array}$ & $\begin{array}{l}\text { Épidémiologie de la maladie et méthodes simples de } \\
\text { lutte antivectorielle d'un bon rapport coût/efficacité. } \\
\text { Nouveaux agents chimiothérapeutiques et meilleur } \\
\text { emploi des médicaments existants. } \\
\text { Tests diagnostiques rapides, sensibles. } \\
\text { Pathogénie de la maladie. }\end{array}$ \\
\hline $\begin{array}{l}\text { Maladie de } \\
\text { Chagas }\end{array}$ & $\begin{array}{l}\text { Maladie de } \\
\text { Chagas }\end{array}$ & $\begin{array}{l}\text { Épidémiologie (EPICHA) } \\
\text { Chimiothérapie (CHEMCHA) } \\
\text { Immunologie (IMMCHA) }\end{array}$ & $\begin{array}{l}\text { Analyse des facteurs de risque hôte/parasite responsa- } \\
\text { bles des différentes formes de la maladie. } \\
\text { Nouveaux médicaments et méthodes de stérilisation du } \\
\text { sang pour transfusion. } \\
\text { Tests diagnostiques améliorés, vaccins. }\end{array}$ \\
\hline Leishmanioses & Leishmanioses & $\begin{array}{l}\text { Épidémiologie (EPILEISH) } \\
\text { Chimiothérapie (CHEMLEISH) } \\
\text { Immunologie (IMMLEISH) }\end{array}$ & $\begin{array}{l}\text { Études approfondies du cycle de transmission. } \\
\text { Identification des hôtes réservoirs de parasites. } \\
\text { Amélioration de la lutte antivectorielle. } \\
\text { Nouveaux médicaments, posologies améliorées. } \\
\text { Vaccins, tests diagnostiques. }\end{array}$ \\
\hline Lèpre & $\begin{array}{l}\text { Immunologie } \\
\text { Chimiothérapie }\end{array}$ & $\begin{array}{l}\text { IMMLEP } \\
\text { THELEP }\end{array}$ & $\begin{array}{l}\text { Vaccins, tests diagnostiques. } \\
\text { Posologies améliorées et nouveaux médicaments. }\end{array}$ \\
\hline $\begin{array}{l}\text { Lutte biologique } \\
\text { contre les } \\
\text { vecteurs }\end{array}$ & $\begin{array}{l}\text { Lutte biologique } \\
\text { contre les } \\
\text { vecteurs }\end{array}$ & $\mathrm{BCV}$ & $\begin{array}{l}\text { Agents biologiques de lutte contre les vecteurs de } \\
\text { maladies. }\end{array}$ \\
\hline $\begin{array}{l}\text { Recherche sociale } \\
\text { et économique }\end{array}$ & $\begin{array}{l}\text { Recherche sociale } \\
\text { et économique }\end{array}$ & SER & $\begin{array}{l}\text { Facteurs sociaux et économiques influençant la trans- } \\
\text { mission de la maladie et l'efficacité de la lutte. }\end{array}$ \\
\hline
\end{tabular}

Le Groupe de travail scientifique sur les sciences biomédicales (BIOS) a été dissous à la fin de 1985. BIOS avait pour tâche d'encourager l'application des progrès effectués en biologie fondamentale à la recherche sur les six maladies, et au renforcement de cette recherche dans les pays tropicaux. Cette dernière activité a maintenant été reprise par le Groupe renforcement de la recherche.

$\mathrm{m} / \mathrm{s} n^{\circ} 10$ vol. 5 , décembre 89 
Les comités d'orientation scientifique sont composés de huit à quinze membres nommés pour une période déterminée, habituellement trois années renouvelables. Des personnalités additionnelles peuvent être cooptées. Ces comités se réunissent au moins une fois par an. Ils planifient les activités, analysent les rapports intérimaires et terminaux des projets et jugent les propositions nouvelles qui leur sont faites. Les critères servant à l'analyse et à la sélection des projets sont: leur concordance avec les priorités et objectifs et, évidemment, le niveau scientifique et la qualité de la présentation du projèt. Le Tableau I (p. 775), repris de «La Science à l'œuvre » (1987), montre la structure des groupes et comités en fonction de chaque maladie ainsi que les principales lignes de recherche.

Lorsque le besoin s'en fait sentir, les comités d'orientation convoquent une réunion de spécialistes de tel ou tel domaine, permettant de revoir les priorités et, éventuellement, d'en fixer de nouvelles.

Outre les propositions qu'il reçoit, analyse, accepte, rejette ou amende, chaque comité d'orientation peut, en fonction de ses priorités, solliciter des projets correspondant à des besoins non encore satisfaits. Les comités ont donc un rôle passif (réception et examen des projets) et actif (prospection et sollicitation des projets). Concernant la durée des projets, elle ne dépasse généralement pas trois années, à l'issue desquelles un rapport final est examiné par le comité d'orientation. Toutefois des projets ponctuels d'une année peuvent être financés par les comités ou directement par le directeur du Programme, en consultation avec le comité correspondant (Fonds d'initiative du directeur : DIF).

La nature et la valeur des projets reçus par TDR sont inégales. S'il est courant et légitime de recevoir de bons projets de recherche fondamentale émanant de laboratoires bien équipés en hommes et matériel basés dans les pays développés, il est en revanche plus difficile et moins fréquent d'obtenir de bons projets de recherche de terrain (épidémiologie, entomologie, sciences sociales...) pro-

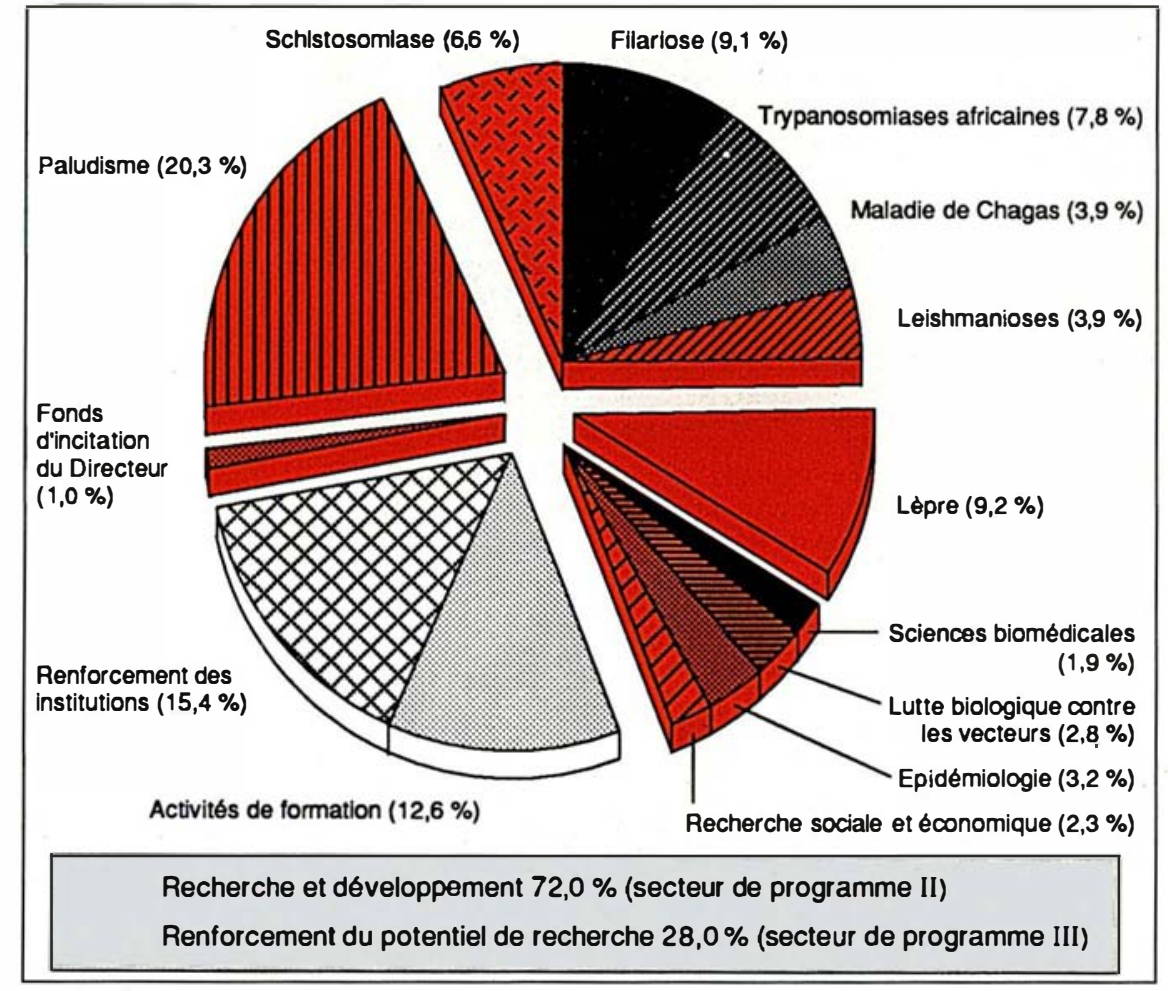

Figure 1. Ventilation des fonds alloués aux projets TDR par composante et secteur de programme, moyenne sur 1975-1988. (TDR: neuvième rapport du programme/179.)

localisés - souvent isolés - dans les pays endémiques.

Bien que ne correspondant pas à la nature et à la vocation tropicale de TDR, ce constat n'est pas paradoxal. La recherche de terrain, effectuée dans des conditions matérielles et intellectuelles souvent pénibles n'attire plus suffisamment de jeunes chercheurs, de plus en plus fascinés - la formation qu'ils acquièrent en est souvent responsable - par les « lumières de la ville » que sont devenues, rapidement, les sciences biologiques modernes. La tendance du Programme est donc, pour redresser cette dérive, d'assister les chercheurs du terrain dans la préparation et la présentation de leurs projets à TDR.

\section{Le groupe de renforcement de la recherche}

Parallèlement, le TDR possède également une structure dont l'objectif est de renforcer les institutions basées dans des zones d'endémies et de permettre ainsi aux chercheurs de mener leur travail là où il doit l'être en priorité : les régions tropicales où ces maladies sévissent. Le RSG (research strengthening group) est ainsi responsable de l'examen des demandes faites par ces instituts. Comme les comités d'orientation, il est composé par des personnalités extérieures assistées du secrétariat. Il reçoit et sélectionne les demandes. Il peut aussi, compte tenu des besoins, se livrer à une prospection sélective tenant compte des priorités de la recherche à effectuer dans tel domaine et dans telle région géographique. Cela se fait par des visites planifiées, par une liaison étroite entre les responsables et souvent, comme pour les projets de recherche, par une assistance du secrétariat de TDR à la mise au point des dossiers à présenter au RSG.

Il va de soi que le renforcement des instituts de recherche ne va pas sans formation de leurs chercheurs. Celle- 
ci, un des points forts de TDR, participe d'une démarche comparable à celle qui prévaut pour les projets de recherches et de renforcement: les dossiers sont reçus par le secrétariat, ils sont évalués par un groupe ad hoc qui, après avoir été composé du secrétariat, sera désormais un sousgroupe du RSG, qui effectue une sélection tenant compte de nombreux facteurs: niveau, institution du candidat, poste occupé au retour, centre de formation, degré de priorité de la recherche envisagée, balance géographique, etc.

Fin 1988, 672 chercheurs avaient bénéficié d'une formation assistée par TDR à tous les niveaux universitaires, depuis les stages spécialisés de courte durée jusqu'à la maîtrise et au doctorat.

Il est à noter que la fuite des cerveaux, si importante ailleurs, est tout à fait exceptionnelle à TDR: les étudiants ou chercheurs qui ne sont pas retournés «au pays » se comptent sur les doigts d'une main. Cela est peut-être dû au suivi permanent qu'assure le Programme à ses étudiants.

Outre le support direct aux boursiers, TDR a également favorisé la création, au sein d'universités localisées en pays endémiques, de centres de formation au niveau du DEA.

C'est ainsi que l'université de Côted'Ivoire a créé, à Bouaké, un Centre de formation en entomologie médicale et vétérinaire (CEMV) qui accueille, depuis 1979, des étudiants de l'ensemble des pays francophones africains. Ce centre, travaillant en étroite collaboration avec les institutions spécialisées de la région africaine et de France (ORSTOM et université de Paris...), assure une formation associant l'éducation et la recherche. Récemment évalué, il peut

être considéré comme un centre d'excellence en ce qui concerne l'entomologie médicale et la lutte contre les vecteurs.

\section{La communication}

TDR bénéficie d'une cellule de communication et d'édition qui produit des séries de documents de nature scientifique ainsi qu'une lettre d'information. Cette cellule a été, depuis peu, renforcée.

\section{La gestion, le budget}

Au quotidien, la gestion de TDR est assurée, au siège de l'OMS à Genève, par un secrétariat composé d'un directeur, des secrétaires des comités d'orientation et du Groupe de renforcement - chercheurs spécialisés dans leurs domaines respectifs - et d'administrateurs. De plus, le Pro-

Le Conseil conjoint de Coordination (JCB), organe directeur suprême du TDR, est constitué de représentants de 30 gouvernements et organisations. Le JCB passe en revue les activités du TDR, examine ses plans à long terme, décide de son budget et évalue son état d'avancement.

Le Comité consultatif scientifique et technique (STAC) se compose de 15 à 18 membres faisant autorité dans des disciplines scientifiques variées. Le STAC examine les activités scientifiques et techniques du TDR et formule des recommandations au JCB et à l'agent d'exécution (OMS).

Le Comité permanent, qui est composé de représentant des trois organisations parrainant de façon conjointe (PNUD, Banque mondiale, OMS) supervise la gestion et les finances du Programme. L'Agent d'exécution chargé du programme est l'Organisation mondiale de la Santé (OMS), qui a son siège à Genève, Suisse.

Les Comités d'orientation gèrent les activités de recherche et développement du TDR. Ils préparent les plans scientifiques, formulent des recommandations quant aux propositions de financement de travaux de recherche, et font le point des résultats obtenus dans le cadre de projets déjà financés. Les secrétaires des comités d'orientation pour la recherche sur les différentes maladies font partie du personnel du TDR, qui travaille directement au sein des divisions ou services compétents de l'OMS afin d'assurer la coordination de la recherche avec les activités nationales et internationales de lutte contre les maladies. Les Groupes de travail scientifiques (SWG) réunissent des spécialistes participant aux activités du TDR afin de faire périodiquement le point sur diverses questions.

Le Groupe Renforcement de la recherche (RSG) se compose de 12 à 15 membres choisis pour leur expérience en gestion de la recherche et de formation à la recherche. Il est chargé de la gestion des efforts du TDR en vue de renforcer le potentiel de recherche des pays en développement dans lesquels les maladies cibles du TDR sont endémiques. gramme/170.) 
gramme est représenté au sein des bureaux régionaux de l'Organisation.

Le budget (63 millions de dollars pour 1988-1989) est ventilé entre la recherche et le développement $(72 \%)$ et le renforcement du potentiel de recherche $(23 \%)$, incluant la formation $(12,6 \%)$. La figure ci-jointe (figure I) présente la distribution détaillée des crédits, eri fonction des composantes, selon les valeurs moyennes de 1975 à 1988 (maladies tropicales, progrès de la recherche internationale, 1987-1988, neuvième rapport du Programme.).

\section{La structure organique}

Sur un plan plus global et politique, la structure de pilotage du Programme apparaît sur la figure 2. Le Programme est en effet soumis à une supervision scientifique du Comité consultatif scientifique et technique (STAC) et gestionnaire et financière du Comité des agences parrainantes (PNUD, Banque mondiale et OMS). L'organisme directeur de TDR est le comité conjoint de coordination (JCB) composé de représentants de 30 gouvernements et organisations.

\section{Conclusions}

Le TDR n'est pas, loin s'en faut, le rédempteur de la recherche en médecine tropicale. La coopération internationale, régionale et bilatérale a fait beaucoup - et depuis longtemps - dans ce domaine. Pour la France, les ministères de la Coopération et de la Recherche, l'ORSTOM, les universités, les instituts Pasteur, dans leurs bases tropicales et en coopération avec les instituts nationaux et régionaux, continuent de travailler dans de nombreux domaines, parallèles à - ou complémentaires de ceux de TDR. Le CRDI canadien, ainsi que les universités en font autant à travers leur assistance à de nombreuses institutions tropicales. Les Commissions européennes, à travers leur DG XII, sont venues plus récemment à la recherche en médecine tropicale, et bénéficient de moyens importants. Il existe d'ailleurs une coopération constante et harmonieuse entre le TDR et l'unité
Le TDR n'est pas non plus, comme on l'entend également, urbi et orbi, ce programme trop riche, prétentieux et agressif que certains décrivent comme un détourneur de fonds qui seraient mieux utilisés ailleurs. Le TDR doit être considéré, de mon point de vue, comme un programme opportun - et non opportuniste qui a permis la consolidation - et non la création - d'un vaste réseau mondial de recherche sur les maladies tropicales. La réunion périodique, au sein d'un même programme, de spécialistes de telle maladie - ou de telle discipline - outre les sommes importantes qui sont distribuées, ont permis une meilleure coordination des activités et une collaboration améliorée entre les institutions et laboratoires du Nord et du Sud. Des fonds importants ont été également dévolus au renforcement des instituts et des hommes dans les zones endémiques, ce qui n'est pas le moindre mérite d'un programme encore jeune et qui n'est certainement pas suffisamment utilisé par les francophones du Nord et du Sud

\section{René Le Berre}

Docteur ès sciences, TDR, Organisation mondiale de la santé, 1211 Genève 27, Suisse.

\section{Summary}

The special programme for research and training in tropical diseases (TDR) : a personal point of view

After presenting the history and structures of TDR, the author concludes that this programme is neither the redeemer of research on tropical diseases nor the pretentious and agressive programme it is sometimes described to be. Rather, from the author's point of view, it is an opportune programme which has permitted the consolidation - not the creation - of a vast global network of research on tropical diseases. 